\title{
Antitumor Agents 259. Design, Syntheses and Structure-Activity- Relationship Study of Desmosdumotin C Analogs
}

\author{
Kyoko Nakagawa-Goto ${ }^{\mathrm{a}}$, Tzu-Hsuan Chen ${ }^{\mathrm{a}}$, Chieh-Yu Peng $^{\mathrm{a}}$, Kenneth F. Bastow ${ }^{\mathrm{b}}$, and Kuo- \\ Hsiung Lee ${ }^{a,{ }^{*}}$ \\ a Natural Products Research Laboratories, School of Pharmacy, University of North Carolina, Chapel Hill, \\ NC 27599, USA \\ b Division of Medicinal Chemistry \& Natural Products, School of Pharmacy, University of North Carolina, \\ Chapel Hill, NC 27599, USA
}

\section{Abstract}

Desmosdumotin C (1) and its analogs previously showed potent, selective in vitro anticancer activity. To explore structure-activity-relationships of $\mathbf{1}$ and further increase potency and selectivity, fifteen novel analogs (7-15 and 21-26) were synthesized, and evaluated for cytotoxity against several human tumor cell lines, as well as inhibition of human endothelial (HUVEC) replication. 4-Bromo-3', 3', 5'tripropyl analog 26 showed significant cytotoxity against A549, A431, 1A9, and HCT-8 with $\mathrm{ED}_{50}$ values of $1.0,1.2,0.9$, and $1.3 \mu \mathrm{g} / \mathrm{mL}$ respectively. Compound $\mathbf{2 6}$ also strongly inhibited the growth of matched tumor cells, KB-VIN and its parent cell KB. Furthermore, analogs $\mathbf{1 3}$ and $\mathbf{2 1}$ were over fivefold more potent against KB-VIN than KB. Bromination of ring-B and tripropyl functionalization of ring-A enhanced activity, while alkylation of ring-B promoted KB-VIN/KB selectivity. 2-Furyl analog 16 showed selective activity against HUVEC, suggesting that it may have potential as a new prototype for angiogenesis inhibition.

\section{Introduction}

Natural products are a significant source of drug candidates. An impressive number of modern drugs have been developed from natural sources, especially from plants used as traditional folk medicines. ${ }^{1}$ Thus, drug discovery from medicinal plants plays an important role in the treatment and prevention of various human diseases, and the continuous importance of natural products in modern medicine has been highlighted in several recent reviews and reports. ${ }^{2-6}$ Our research interest is the discovery and development of novel anticancer drugs from natural plants. Currently, about three-quarters of anticancer drugs come from either natural products or their derivatives. ${ }^{7}$ Cancer is a leading cause of death worldwide accounting for thirteen percent of all deaths in 2005,8 even though many effective and diverse cancer treatments have been approved and are used. Major problems associated with cancer chemotherapy include undesirable toxic side effects and multidrug resistance. Therefore, a pressing need to develop more effective antitumor drugs still remains.

Desmos dumosus (Roxb.) is a climber plant found in alluvial forests in southern Asia, and has been used in Chinese folk medicine as an antimalarial, insecticidal, antirheumatic, antispasmodic, and analgesic agent. ${ }^{9}$ Recently, some novel bioactive flavonoids, named desmosdumotins B and C, were isolated from the plant root. ${ }^{10,11}$ Desmosdumotin $\mathrm{C}(\mathbf{1})$ has a distinctive chalcone skeleton with an unusual non-aromatic A ring possessing a gem-dimethyl 
group on C-3' and methyl group on C-5'. It showed significant and selective in vitro cytotoxicity against $1 \mathrm{~A} 9$ (ovarian cancer) and A549 (human lung carcinoma) cell lines with $\mathrm{IC}_{50}$ values of 4.0 and $3.5\left(\mu \mathrm{g} / \mathrm{mL}\right.$, respectively. ${ }^{10}$ In addition, it was more active against drug-resistant KBVIN cells than against the parent KB (epidermoid nasopharyngeal carcinoma) cell line. Thus, $\mathbf{1}$ is a promising new lead for further new antitumor analog development. We previously achieved a simple first total synthesis of $\mathbf{1}^{11}$ as well as some modifications of the A- and Brings and reported the cytotoxic activity data against four tumor cell lines, 1A9, A549, KB and KB-VIN. ${ }^{12}$ Among the tested compounds, 4-bromodesmosdumotin C (2) showed two- to three-fold enhanced activity compared with $\mathbf{1}$. This promising result encouraged us to continue the modification of this series to develop novel anticancer drug candidates. In this paper, we describe further modifications of the A- and B-rings as well as evaluation of newly and previously synthesized analogs against seven human tumor cell lines, A549, 1A9, KB, KBVIN, A431 (epidermoid skin carcinoma), HCT-8 (colon adenocarcinoma), and PC-3 (prostate cancer), as well as HUVEC.

\section{Chemistry}

The simplicity of our accomplished synthesis ${ }^{11}$ of $\mathbf{1}$ allows easy modification of the A-ring, by using another alkyl iodide rather than methyl iodide in the first step, and the B-ring, by using a different aromatic aldehyde from benzaldehyde in the final step (Scheme 1). First, nine B-ring modified analogs, 7-15, were synthesized from intermediate 29 using 4fluorophenylaldehyde, $o$ - and $p$-tolualdehyde, 4-ethylbenzaldehyde, 2,6- and 3,5dimethylbenzaldehyde, 2,4,6-trimethylbenzaldehyde, 2-vinylbenzaldehyde, and 1naphthaldehyde under basic aldol conditions. Second, modification of the A-ring to introduce an ethyl or propyl group at the $\mathrm{C}-3$ and $\mathrm{C}^{\prime}-5^{\prime}$ positions was accomplished by the following method. Treatment of trihydroxyacetophenone $\mathbf{2 8}$ with 3 mol eq. of ethyl or propyl iodide in the presence of sodium methoxide provided the corresponding trialkylacetophenones ( $\mathbf{3 2}$ and $\mathbf{3 3}$, respectively). Similarly, treatment of $\mathbf{3 0}^{12}$ with 1 mol eq. of ethyl iodide afforded $\mathbf{3 1}$. Selective methylation of the resulting trialkylacetophenones, 31-33, with an excess of $\mathrm{TMSCHN}_{2}$ at low temperature, followed by aldol reaction with benzaldehyde gave $\mathbf{2 1}, \mathbf{2 2}$, and $\mathbf{2 5}$. The aldol reaction of $\mathbf{3 5}$ and $\mathbf{3 6}$ with 4-methyl, 4-ethyl, or 4-bromobenzaldehyde gave Aand B-ring analogs, 23, 24, and 26, respectively. All synthesized compounds, except for $\mathbf{7}$, $\mathbf{1 2}$, and $\mathbf{1 5}$, exist as a mixture of two tautomeric isomers as discussed in our prior papers. ${ }^{12}$ 13 Compounds 1-6,16-19, 20 and 27 were synthesized previously. 12

\section{Results and Discussion}

Together with 1 and previously synthesized analogs, all newly synthesized compounds were evaluated for in vitro anticancer activity against several human tumor cell lines, A549, A431, 1A9, HCT-8, PC-3, KB and KB-VIN, and against HUVEC, a normal cell useful for assessing anti-angiogenic potential. The average $\mathrm{ED}_{50}$ values $(\mu \mathrm{g} / \mathrm{mL})$ are listed in Table 1.

Compound 1 showed activity against A549, 1A9, HCT-8 and HUVEC with ED $_{50}$ values of $3.5,3.5,3.7$, and $2.1 \mu \mathrm{g} / \mathrm{mL}$, respectively, but was less active against $\mathrm{A} 431$ and $\mathrm{PC}-3$ cells. Compound 1 was also 1.3-fold more active against KB-VIN cells $\left(\mathrm{ED}_{50} 3.0 \mu \mathrm{g} / \mathrm{mL}\right)$ than the parent KB cell line. Except for 14, 25 and 26, most 1-analogs showed similar activity patterns, as described later.

Significantly enhanced cytotoxic activities were observed with 2, 2-vinyl desmosdumotin C (14), 3', 3', 5'-tripropyl desmosdumotin C (25), and 4-bromo-3', 3',5'-tripropyl desmosdumotin $\mathrm{C}$ (26). Especially, $\mathbf{2 6}$ displayed enhanced in vitro antitumor activity against all cell lines with $\mathrm{ED}_{50}$ values of $0.9-2.3 \mu \mathrm{g} / \mathrm{mL}$. In addition, 26 was not affected by multidrug resistant expressing P-glycoprotein, since it showed similar and potent cell growth inhibition of KB and 
KB-VIN cell replication ( $\mathrm{ED}_{50} 0.9 \mu \mathrm{g} / \mathrm{mL}$ ). The strong activity of $\mathbf{2 6}$ could be predicted since it combines the structural features of 4-bromo 2 and $3^{\prime}, 3^{\prime}, 5^{\prime}$-tripropyl $\mathbf{2 5}$, which also were quite potent against A549, 1A9, KB, and KB-VIN cell lines. Notably, 25 strongly inhibited the growth of $\mathrm{KB}$ and $\mathrm{KB}-\mathrm{VIN}$ cells with $\mathrm{ED}_{50}$ values of 0.6 and $0.8 \mu \mathrm{g} / \mathrm{mL}$, respectively.

Interestingly, while most analogs, including 2 , were generally less active against PC-3 replication, three compounds, $\mathbf{1 4}, \mathbf{2 5}$ and $\mathbf{2 6}$, displayed enhanced activity against PC-3 with $\mathrm{ED}_{50}$ values of $4.6,3.3$ and $2.3 \mu \mathrm{g} / \mathrm{mL}$, respectively. These three analogs also inhibited the growth of A431 cells with $\mathrm{ED}_{50}$ values of 2.6, 1.9 and $1.2 \mu \mathrm{g} / \mathrm{mL}$, respectively. Furthermore, in the PC-3, A431, and HCT-8 cell lines, bromination at C-4 (2) decreased cytotoxic activity relative to $\mathbf{1}$, while fluorination (7) increased activity.

From comparison of the data for $\mathbf{1}$ with that of 3-5 and 8-9, the insertion of a methoxy or methyl group on the phenyl B-ring had either no effect on or slightly decreased activity, regardless of the substitution position. The 4-ethyl (10), 2,6-dimethyl (11), and trimethyl (13) derivatives also showed decreased activity against all cell lines, although $\mathbf{1}$ and the 3,5dimethyl derivative (12) had comparable potencies. However, it is noteworthy that the introduction of an alkyl group on the B-ring enhanced the growth inhibition of the KB-VIN cell line resulting in a two- to five-fold ratio of KB/KB-VIN selectivity (see 8-14). However, the insertion of hetero aromatic ring rather than the phenyl ring did not affect the cytotoxic activity (see 16-18).

For the A-ring analogs, tetramethyl analog 19, dimethyl analog 20 (ceroptene), ${ }^{14} 3^{\prime}, 3^{\prime}$ dimethyl-5'-ethyl analog 21, and 4'-demethyl analog 27 were less potent against all cell lines than the parent compound $\mathbf{1}$. However, $\mathbf{2 1}$ showed a remarkably high KB/KB-VIN selectivity ratio of 14.3. Among the triethyl compounds, $\mathbf{2 3}$ and $\mathbf{2 4}$ were less active than $\mathbf{1}$, while $\mathbf{2 2}$, without a substituent on the B-ring, showed slightly enhanced activity against most cell lines, except for A549 and A431.

Angiogenesis is necessary for tumor growth and metastasis; therefore, it presents another target for cancer treatment. Selected compounds were also evaluated in a standard anti-angiogenesis assay using HUVEC. All tested compounds, except 20, showed potent activity with $\mathrm{ED}_{50}$ values less than $4.0 \mu \mathrm{g} / \mathrm{mL}$. Compound $\mathbf{1 4}$ showed the highest potency with an $\mathrm{ED}_{50}$ value of $1.1 \mu \mathrm{g} / \mathrm{mL}$. However, this compound also showed strong activity against other tumor cell lines suggesting that the activity against HUVEC may be non-specific. However, analogs 16-18, in which the phenyl B-ring was replaced with 5-membered heteroaromatic rings, demonstrated significant activity against HUVEC with less activity against tumor cell replication. In particular, 2-furyl analog 16 possessed the highest differential with an $\mathrm{ED}_{50}$ value of $2.4 \mu \mathrm{g} /$ $\mathrm{mL}$ against HUVEC compared to $\mathrm{ED}_{50}$ values of $7.9-20.6 \mu \mathrm{g} / \mathrm{mL}$ against the other tumor cell lines. This selective activity suggests that $\mathbf{1 6}$ may have potential as a new prototype as an angiogenesis inhibitor.

In summary, the preliminary SAR studies led to the following observations: 1) a bromide function on the B-ring enhanced activity ( $\mathbf{1}$ vs $\mathbf{2}$ and $\mathbf{2 5}$ vs $\mathbf{2 6}) ; 2$ ) the order of potency for C-3' and $-5^{\prime}$ functionalities was $3^{\prime}, 3^{\prime}, 5^{\prime}$-tripropyl $>3^{\prime}, 3^{\prime}, 5^{\prime}$-trimethyl $>3^{\prime}, 3^{\prime}, 5^{\prime}$-triethyl $>3^{\prime}, 3^{\prime}$ dimethyl-5'-ethyl > 3', $3^{\prime}$-dimethyl > 3', $3^{\prime}, 5^{\prime}, 5^{\prime}$-tetramethyl; 3) an alkyl group on the B-ring promoted KB-VIN/KB selectivity; 4) a C-4'methoxy group is required for activity; 5) 5membered hetero B-rings increased the differential angiogenesis activity. From all results, 26 showed significant promise as a new antitumor drug candidate because of its potent cytotoxity against tumor cell lines and lack of multidrug cross resistance. Focused studies on the KB-VIN/KB selectivity of desmosdumotin series are currently ongoing and the results will be reported elsewhere in the near future. 


\section{Experimental Section}

\section{Materials and Methods}

Melting points were measured with a Fisher Johns melting apparatus without correction. Proton nuclear magnetic resonance $\left({ }^{1} \mathrm{H}\right.$ NMR) spectra were measured on a $300 \mathrm{MHz}$ Varian Gemini 2000 spectrometer using TMS as internal standard. All chemical shifts are reported in ppm. Mass spectra were measured on a PE-SCIEX API 3000 instrument with turbo ion spray source or Agilent-1100, LC/MSD-Trap. Thin-layer chromatography (TLC) and preparative TLC were performed on precoated silica gel GF plates purchased from Merck, Inc. Biotage Flash ${ }^{\mathrm{TM}}$ or Isco Companion systems were used for medium pressure column chromatography. Silica gel (200-400 mesh) from Aldrich, Inc. was used for column chromatography. All other chemicals were obtained from Aldrich, Inc.

\section{General Procedures for the Aldol Reactions}

A solution of acetyl compound (29 or 34-36) in EtOH-50\% aq. KOH (1:1, v/v) and an appropriate aldehyde (excess) was stirred at room temperature. After the reaction was complete by TLC analysis, the mixture was poured into ice-cold $1 \mathrm{~N} \mathrm{HCl}$, then extracted with $\mathrm{CH}_{2} \mathrm{Cl}_{2}$. The extract was washed with brine, dried over $\mathrm{Na}_{2} \mathrm{SO}_{4}$, and concentrated in vacuo. The residue was chromatographed on silica gel with $\mathrm{CH}_{2} \mathrm{Cl}_{2}$-hexane as eluent to afford the target compound, which was crystallized from $\mathrm{CH}_{2} \mathrm{Cl}_{2}$-hexane.

\section{4-Fluoro desmosdumotin C (7)}

Yellow prisms, mp $109-110{ }^{\circ} \mathrm{C}\left(\mathrm{CH}_{2} \mathrm{Cl}_{2}\right.$-hexane). ${ }^{1} \mathrm{H}-\mathrm{NMR}\left(\mathrm{CDCl}_{3}\right): \delta 19.18$ (s, $1 \mathrm{H}$, chelated$\mathrm{OH}), 8.27(\mathrm{~d}, 1 \mathrm{H}, J=15.6 \mathrm{~Hz}$, trans-olefinic proton), $7.89(\mathrm{~d}, 1 \mathrm{H}, J=15.6 \mathrm{~Hz}$, trans-olefinic proton), 7.71-7.60 (m, 2H, Ar-2,6- H), ), 7.12-7.00 (m, 2H, Ar-3,5-H), $3.96\left(\mathrm{~s}, 3 \mathrm{H}, \mathrm{OCH}_{3}\right)$, $2.00\left(\mathrm{~s}, 3 \mathrm{H}, 5^{\prime}-\mathrm{CH}_{3}\right), 1.38\left(\mathrm{~s}, 6 \mathrm{H}, 3^{\prime}-\mathrm{CH}_{3} \times 2\right)$. MS m/z $331\left(\mathrm{M}^{+}+1\right)$. Anal. $\left(\mathrm{C}_{19} \mathrm{H}_{19} \mathrm{FO}_{4}\right) \mathrm{C}, \mathrm{H}$, $\mathrm{O}$.

\section{4-Methyl desmosdumotin C (8)}

Yellow prisms, mp $110-111^{\circ} \mathrm{C}\left(\mathrm{CH}_{2} \mathrm{Cl}_{2}\right.$-hexane). ${ }^{1} \mathrm{HNMR}$ (300 $\left.\mathrm{MHz}, \mathrm{CDCl}_{3}\right): \delta=19.32$ and 18.92 (3:1, each s, $1 \mathrm{H}$, chelated-OH), 8.52 and $8.31(1: 3$, each d, $1 \mathrm{H}, J=15.7 \mathrm{~Hz}$, olefin), 7.95 and $7.93\left(1: 3\right.$, each d, $1 \mathrm{H}, J=15.7 \mathrm{~Hz}$, olefin), 7.62-7.54 (m, 2H, Ar-2', $\left.6^{\prime}-H\right), 7.24-7.17(\mathrm{~m}$, 2H, Ar-3, 5- H), 3.96 and 3.89 (3:1, each s, 3H, $\left.\mathrm{OCH}_{3}\right), 2.00$ and 1.96 (3:1, each s, 3H, 5'$\left.\mathrm{CH}_{3}\right), 1.47$ and $1.38\left(1: 3\right.$, each s, $\left.6 \mathrm{H}, 3^{\prime}-\mathrm{CH}_{3} \times 2\right)$. $\mathrm{MS} m / z 327\left(\mathrm{M}^{+}+1\right)$. Anal. $\left(\mathrm{C}_{20} \mathrm{H}_{22} \mathrm{O}_{4}\right) \mathrm{C}$, $\mathrm{H}, \mathrm{O}$.

\section{2-Methyl desmosdumotin C (9)}

Yellow prisms, mp 93-94 ${ }^{\circ} \mathrm{C}\left(\mathrm{CH}_{2} \mathrm{Cl}_{2}\right.$-hexane). ${ }^{1} \mathrm{H}$ NMR (300 MHz, $\left.\mathrm{CDCl}_{3}\right): \delta=19.32$ and $18.62(4: 1$, each s, $1 \mathrm{H}$, chelated-OH), $8.34(\mathrm{~s}, 2 \mathrm{H}), 8.05-7.94(\mathrm{~m}, 1 \mathrm{H}), 7.51-7.25(\mathrm{~m}, 3 \mathrm{H}, \mathrm{Ar}-$ $H$ ), 4.10 and $4.03\left(4: 1\right.$, each s, $\left.3 \mathrm{H}, \mathrm{OCH}_{3}\right), 2.63\left(\mathrm{~s}, 3 \mathrm{H}, \mathrm{Ar}-\mathrm{CH}_{3}\right), 2.15$ and 2.01 (4:1, each s, $\left.3 \mathrm{H}, 5^{\prime}-\mathrm{CH}_{3}\right), 1.52\left(\mathrm{~s}, 6 \mathrm{H}, 3^{\prime}-\mathrm{CH}_{3} \times 2\right)$. MS m/z $327\left(\mathrm{M}^{+}+1\right)$. Anal. $\left(\mathrm{C}_{20} \mathrm{H}_{22} \mathrm{O}_{4}\right) \mathrm{C}, \mathrm{H}, \mathrm{O}$.

\section{4-Ethyl desmosdumotin C (10)}

Yellow prisms, mp 90-91 ${ }^{\circ} \mathrm{C}\left(\mathrm{CH}_{2} \mathrm{Cl}_{2}\right.$-hexane). ${ }^{1} \mathrm{H}$ NMR (300 MHz, $\left.\mathrm{CDCl}_{3}\right): \delta 19.19$ and $18.80(2: 1$, each $\mathrm{s}, 1 \mathrm{H}$, chelated-OH), 8.52 and $8.32(1: 2$, each $\mathrm{d}, 1 \mathrm{H}, J=15.9 \mathrm{~Hz}$, olefin), 7.96 and $7.94(1: 2$, each d, $1 \mathrm{H}, J=15.9 \mathrm{~Hz}$, olefin), 7.62 and $7.60(1: 2$, each d, $2 \mathrm{H}, J=7.9 \mathrm{~Hz}, \mathrm{Ar}-2$, 6-H), 7.23 and $7.22(1: 2$, each d, $2 \mathrm{H}, J=7.9 \mathrm{~Hz}, \mathrm{Ar}-3,5-H), 3.95$ and $3.89(2: 1$, each s, $3 \mathrm{H}$, $\left.\mathrm{OCH}_{3}\right), 2.68\left(\mathrm{q}, 2 \mathrm{H}, J=7.7 \mathrm{~Hz}, \mathrm{Ar}-\mathrm{CH}_{2} \mathrm{CH}_{3}\right), 2.00$ and 1.96 (2:1. each s, $\left.3 \mathrm{H}, 5^{\prime}-\mathrm{CH}_{3}\right), 1.47$ and $1.38\left(1: 2\right.$, each s, $\left.6 \mathrm{H}, 3^{\prime}-\mathrm{CH}_{3} \times 2\right), 1.25\left(\mathrm{t}, 3 \mathrm{H}, J=7.7 \mathrm{~Hz}, \mathrm{Ar}_{-} \mathrm{CH}_{2} \mathrm{CH}_{3}\right)$. MS m/z 339 $\left(\mathrm{M}^{+}-1\right)$. Anal. $\left(\mathrm{C}_{21} \mathrm{H}_{24} \mathrm{O}\right) \mathrm{C}, \mathrm{H}, \mathrm{O}$. 


\section{2,6-Dimethyl desmosdumotin C (11)}

Yellow oil. ${ }^{1} \mathrm{H}$ NMR $\left(300 \mathrm{MHz}, \mathrm{CDCl}_{3}\right): \delta 19.14$ and $18.80(2: 1$, each s, $1 \mathrm{H}$, chelated-OH), $8.17(\mathrm{~d}, 1 \mathrm{H}, J=15.7 \mathrm{~Hz}$, olefin), 7.87 (d, $1 \mathrm{H}, J=15.7 \mathrm{~Hz}$, olefin), 7.20-7.01 (m, 3H, Ar-3,4,5$H$ ), 3.95 and 3.88 (2:1, each s, $\left.3 \mathrm{H}, \mathrm{OCH}_{3}\right), 2.44$ (s, 6H, Ar-2,6- $\left.\mathrm{CH}_{3}\right), 2.00$ and 1.92 (2:1, each $\left.\mathrm{s}, 3 \mathrm{H}, 5^{\prime}-\mathrm{CH}_{3}\right), 1.48$ and $1.35\left(1: 2\right.$, each s, $\left.6 \mathrm{H}, 3^{\prime}-\mathrm{CH}_{3} \times 2\right)$. MS m/z $341\left(\mathrm{M}^{+}+1\right)$. Anal. $\left(\mathrm{C}_{21} \mathrm{H}_{24} \mathrm{O}_{4}\right) \mathrm{C}, \mathrm{H}, \mathrm{O}$.

\section{3,5-Dimethyl desmosdumotin C (12)}

Yellow prisms, mp $105-106{ }^{\circ} \mathrm{C}\left(\mathrm{CH}_{2} \mathrm{Cl}_{2}\right.$-hexane). ${ }^{1} \mathrm{H}$ NMR (300 MHz, $\left.\mathrm{CDCl}_{3}\right): \delta 19.20$ (s, $1 \mathrm{H}$, chelated-OH), 8.31 (d, $1 \mathrm{H}, J=15.7 \mathrm{~Hz}$, olefin), 7.91 (d, $1 \mathrm{H}, J=15.7 \mathrm{~Hz}$, olefin), 7.30 (s, 2H, Ar-2', 6'-H), 7.03 (s, $1 \mathrm{H}, \mathrm{Ar}-4-H), 3.96$ (s, 3H, OCH $\left.H_{3}\right), 2.34$ (s, 6H, Ar-3,5-CH3), 2.00 (s, $\left.3 \mathrm{H}, 5^{\prime}-\mathrm{CH}_{3}\right), 1.39\left(\mathrm{~s}, 6 \mathrm{H}, 3^{\prime}-\mathrm{CH}_{3} \times 2\right)$. MS m/z $341\left(\mathrm{M}^{+}+1\right)$. Anal. $\left(\mathrm{C}_{21} \mathrm{H}_{24} \mathrm{O}_{4}\right) \mathrm{C}, \mathrm{H}, \mathrm{O}$.

\section{2,4,6-Trimethyl desmosdumotin C (13)}

Yellow prisms, mp 87-88 ${ }^{\circ} \mathrm{C}$ (AcOEt-hexane). ${ }^{1} \mathrm{H}$ NMR (300 MHz, CDC1 $\left.1_{3}\right): \delta 19.17$ and $18.48(3: 1$, each s, $1 \mathrm{H}$, chelated-OH), 8.21-8.09 (m, $1 \mathrm{H}$, olefin), 7.96 and 7.90 (1:3, each d, $1 \mathrm{H}, J=15.5 \mathrm{~Hz}$, olefin), 6.92 and $6.90(1: 3$, each s, $2 \mathrm{H}, \mathrm{Ar}-3,5-H), 3.95$ and 3.88 (3:1, each s, $\left.3 \mathrm{H}, \mathrm{OCH}_{3}\right), 2.43$ and 2.41 (3:1, each s, $\left.6 \mathrm{H}, \mathrm{Ar}-2,6-\mathrm{CH}_{3}\right), 2.30$ and $2.29(1: 3$, each s, $3 \mathrm{H}$, Ar-4-CH $\left.\mathrm{CH}_{3}\right), 2.00$ and 1.92 (3:1. each s, 3H, 5'- $\left.\mathrm{CH}_{3}\right), 1.47$ and $1.35\left(1: 3\right.$, each s, $\left.6 \mathrm{H}, 3^{\prime}-\mathrm{CH}_{3} \times 2\right)$. MS m/z $355\left(\mathrm{M}^{+}+1\right)$. Anal. $\left(\mathrm{C}_{22} \mathrm{H}_{26} \mathrm{O}_{4} \cdot 1 / 8 \mathrm{H}_{2} \mathrm{O}\right) \mathrm{C}, \mathrm{H}$.

\section{3-Vinyl desmosdumotin C (14)}

Yellow prisms, mp $76-77{ }^{\circ} \mathrm{C}\left(\mathrm{CH}_{2} \mathrm{Cl}_{2}\right.$-hexane). ${ }^{1} \mathrm{H}$ NMR (300 MHz, $\left.\mathrm{CDCl}_{3}\right): \delta 19.19$ and 18.79 (3:1, each s, $1 \mathrm{H}$, chelated-OH), 8.54 and $8.34(1: 3$, each d, $1 \mathrm{H}, J=15.9 \mathrm{~Hz}$, olefin), 7.96 and $7.93(1: 3$, each d, $1 \mathrm{H}, J=15.9 \mathrm{~Hz}$, olefin), 7.67 and 7.65 (1:3, each br s, $1 \mathrm{H}, \mathrm{Ar}-2-\mathrm{H}), 7.60$ and $7.58(1: 3$, each d, $1 \mathrm{H}, J=7.4 \mathrm{~Hz}, \mathrm{Ar}-4$ or $6-H), 7.47$ and $7.45(1: 3$, each d, $1 \mathrm{H}, J=7.4 \mathrm{~Hz}$, Ar-4 or 6-H), 7.37 and $7.36(1: 3$, each t, $1 \mathrm{H}, \mathrm{Ar}-5-H), 6.74(\mathrm{dd}, 1 \mathrm{H}, J=10.8$ and $17.4 \mathrm{~Hz}$, Ar$\left.\mathrm{CH}=\mathrm{CH}_{2}\right), 5.81\left(\mathrm{~d}, 1 \mathrm{H}, J=17.4 \mathrm{~Hz}, \mathrm{Ar}-\mathrm{CH}=\mathrm{CH}_{2}\right), 5.31\left(\mathrm{~d}, 1 \mathrm{H}, J=10.8 \mathrm{~Hz}, \mathrm{Ar}-\mathrm{CH}=\mathrm{CH}_{2}\right)$, 3.96 and 3.89 ( $3: 1$, each s, $\left.3 \mathrm{H}, \mathrm{OCH}_{3}\right), 2.00$ and 1.96 ( $3: 1$, each s, $\left.3 \mathrm{H}, 5^{\prime}-\mathrm{CH}_{3}\right), 1.48$ and 1.39 (1:3, each s, $\left.6 \mathrm{H}, 3^{\prime}-\mathrm{CH}_{3} \times 2\right)$. MS m/z $359\left(\mathrm{M}^{+}+1\right)$. Anal. $\left(\mathrm{C}_{21} \mathrm{H}_{22} \mathrm{O}_{4}\right) \mathrm{C}, \mathrm{H}, \mathrm{O}$.

\section{2-[1-Hydroxy-3-(naphthalen-1-yl)allyidene]-5-methoxy-4,6,6-trimethylcyclohex-4-ene-1,3- dione (15)}

Yellow prisms, mp $128-129^{\circ} \mathrm{C}\left(\mathrm{CH}_{2} \mathrm{Cl}_{2}\right.$-hexane). ${ }^{1} \mathrm{H} \mathrm{NMR}\left(300 \mathrm{MHz}, \mathrm{CDCl}_{3}\right)$ : $\delta 19.2(\mathrm{~s}, 1 \mathrm{H}$, chelated-OH), $8.83(\mathrm{~d}, 1 \mathrm{H}, J=15.4 \mathrm{~Hz}$, olefin), $8.42(\mathrm{~d}, 1 \mathrm{H}, J=15.4 \mathrm{~Hz}$, olefin), $8.28(\mathrm{~d}, 1 \mathrm{H}$, $J=7.5 \mathrm{~Hz}$, naphthyl-2 or 8-H), 8.04 (d, $1 \mathrm{H}, J=7.5 \mathrm{~Hz}$, naphthyl-2 or 8-H), 7.96-7.84 (m, 2H, naphthyl- $H$ ), 7.64-7.46 (m, 3H, naphthyl-H), 7.37 and 7.36 (1:3, each t, $1 \mathrm{H}, \mathrm{Ar}-5-H), 6.74$ (dd, $1 \mathrm{H}, J=10.8$ and $\left.17.4 \mathrm{~Hz}, \mathrm{Ar}-\mathrm{CH}=\mathrm{CH}_{2}\right), 5.81\left(\mathrm{~d}, 1 \mathrm{H}, J=17.4 \mathrm{~Hz}, \mathrm{Ar}-\mathrm{CH}=\mathrm{CH}_{2}\right), 5.31(\mathrm{~d}, 1 \mathrm{H}$, $\left.J=10.8 \mathrm{~Hz}, \mathrm{Ar}-\mathrm{CH}=\mathrm{CH}_{2}\right), 3.96$ and $3.89\left(3: 1\right.$, each s, $\left.3 \mathrm{H}, \mathrm{OCH}_{3}\right), 2.00$ and 1.96 (3:1, each s, $\left.3 \mathrm{H}, 5^{\prime}-\mathrm{CH}_{3}\right), 1.48$ and $1.39\left(1: 3\right.$, each s, $\left.6 \mathrm{H}, 3^{\prime}-\mathrm{CH}_{3} \times 2\right)$. MS m/z $363\left(\mathrm{M}^{+}+1\right)$. Anal. $\left(\mathrm{C}_{23} \mathrm{H}_{22} \mathrm{O}_{4}\right) \mathrm{C}, \mathrm{H}, \mathrm{O}$.

\section{3',3'-Dimethyl-5'-ethyl desmosdumotin C (21)}

75\% Yield. Yellow oil. ${ }^{1} \mathrm{H}$ NMR (300 MHz, $\left.\mathrm{CDCl}_{3}\right): \delta 19.37$ and $18.90(2: 1$, each s, $1 \mathrm{H}$, chelated-OH), 8.67 and $8.47(1: 2$, each d, $1 \mathrm{H}, J=15.9 \mathrm{~Hz}$, olefin), 8.10 and 8.07 (1:3, each d, $1 \mathrm{H}, J=15.9 \mathrm{~Hz}$, olefin), 7.89-7.76 (m, 2H, Ar-2,6-H), 7.59-7.48 (m, 3H, Ar-3,4,5-H), 4.12 and $4.06\left(2: 1\right.$, each s, $\left.3 \mathrm{H}, \mathrm{OCH}_{3}\right), 2.65$ and $2.62\left(2: 1\right.$, each q, $\left.J=7.4 \mathrm{~Hz}, 2 \mathrm{H}, 5^{\prime}-\mathrm{CH}_{2} \mathrm{CH}_{3}\right)$, 1.62 and $1.53\left(1: 2\right.$, each s, 6H, $\left.3^{\prime}-\mathrm{CH}_{3} \times 2\right), 1.32$ and $1.29\left(2: 1\right.$, each t, $J=7.4 \mathrm{~Hz}, 3 \mathrm{H}, 5^{\prime}-$ $\left.\mathrm{CH}_{2} \mathrm{CH}_{3}\right)$. MS m/z $325\left(\mathrm{M}^{+}-1\right)$. Anal. $\left(\mathrm{C}_{20} \mathrm{H}_{22} \mathrm{O}_{4}\right) \mathrm{C}, \mathrm{H}, \mathrm{O}$. 


\section{3',3',5'-Triethyl desmosdumotin C (22)}

57\% Yield. Yellow oil. ${ }^{1} \mathrm{H}$ NMR (300 MHz, $\left.\mathrm{CDCl}_{3}\right): \delta 19.30$ and $18.89(3: 2$, each s, $1 \mathrm{H}$, chelated-OH), 8.52 and $8.44(2: 3$, each d, $1 \mathrm{H}, J=15.3 \mathrm{~Hz}$, olefin), 7.96 and 7.93 (2:3, each d, $1 \mathrm{H}, J=15.3 \mathrm{~Hz}$, olefin), 7.73-7.66 (m, 2H, Ar-2,6-H), 7.43-7.36 (m, 3H, Ar-3,4,5-H), 4.03 and $3.96\left(3: 2\right.$, each s, $\left.3 \mathrm{H}, \mathrm{OCH}_{3}\right), 2.60$ and $2.56\left(3: 2\right.$, each q, $\left.2 \mathrm{H}, J=7.4 \mathrm{~Hz}, 5^{\prime}-\mathrm{CH}_{2} \mathrm{CH}_{3}\right)$, 2.04-1.76 (m, 4H, 3'- $\left.\mathrm{CH}_{2} \mathrm{CH}_{3} \times 2\right), 1.20$ and $1.16\left(3: 2\right.$, each t, $\left.3 \mathrm{H}, J=7.4 \mathrm{~Hz}, 5^{\prime}-\mathrm{CH}_{2} \mathrm{CH}_{3}\right)$, 0.71 and $0.70\left(2: 3\right.$, each t, $\left.3 \mathrm{H}, J=7.4 \mathrm{~Hz}, 3^{\prime}-\mathrm{CH}_{2} \mathrm{CH}_{3} \times 2\right)$. $\mathrm{MS} \mathrm{m} / z 355\left(\mathrm{M}^{+}+1\right)$. Anal. $\left(\mathrm{C}_{22} \mathrm{H}_{26} \mathrm{O}_{4}\right) \mathrm{C}, \mathrm{H}, \mathrm{O}$.

\section{4-Methyl-3',3',5'-triethyl desmosdumotin C (23)}

63\% Yield. Yellow oil. ${ }^{1} \mathrm{H}$ NMR (300 MHz, $\left.\mathrm{CDCl}_{3}\right): \delta 19.30$ and $18.91(3: 2$, each s, $1 \mathrm{H}$, chelated-OH), 8.50 and 8.41 (2:3, each d, $1 \mathrm{H}, J=15.3 \mathrm{~Hz}$, olefin), 7.96 and 7.93 (2:3, each d, $1 \mathrm{H}, J=15.3 \mathrm{~Hz}$, olefin), 7.64-7.55 (m, 2H, Ar-2,6-H), 7.25-7.14 (m, 2H, Ar-3,5-H), 4.03 and $3.95\left(3: 2\right.$, each s, $\left.3 \mathrm{H}, \mathrm{OCH}_{3}\right), 2.60$ and $2.56\left(3: 2\right.$, each q, $\left.2 \mathrm{H}, J=7.4 \mathrm{~Hz}, 5^{\prime}-\mathrm{CH}_{2} \mathrm{CH}_{3}\right), 2.38$ and 2.35 (3:2, each s, 3H, Ar-4-CH $\left.\mathrm{CH}_{3}\right), 2.07-1.90$ and 1.90-1.72 (3:2, each m, 4H, 3'$\left.\mathrm{CH}_{2} \mathrm{CH}_{3} \times 2\right), 1.30-1.21\left(\mathrm{~m}, 3 \mathrm{H}, 5^{\prime}-\mathrm{CH}_{2} \mathrm{CH}_{3}\right), 0.70\left(\mathrm{t}, 6 \mathrm{H}, J=7.4 \mathrm{~Hz}, 3^{\prime}-\mathrm{CH}_{2} \mathrm{CH}_{3} \times 2\right)$. MS m/ z $367\left(\mathrm{M}^{+}-1\right)$. Anal. $\left(\mathrm{C}_{22} \mathrm{H}_{24} \mathrm{O}_{4}\right) \mathrm{C}, \mathrm{H}$.

\section{4-Methyl-3',3',5'-triethyl desmosdumotin C (24)}

$61 \%$ Yield. Yellow oil. ${ }^{1} \mathrm{H}$ NMR (300 MHz, $\left.\mathrm{CDCl}_{3}\right): \delta 19.30$ and $18.93(2: 1$, each s, $1 \mathrm{H}$, chelated-OH), 8.50 and $8.42(1: 2$, each d, $1 \mathrm{H}, J=15.3 \mathrm{~Hz}$, olefin), 7.97 and 7.93 (1:2, each d, $1 \mathrm{H}, J=15.3 \mathrm{~Hz}$, olefin), 7.68-7.56 (m, 2H, Ar-2,6-H), 7.30-7.18 (m, 2H, Ar-3,5-H), 4.03 and 3.95 (2:1, each s, $\left.3 \mathrm{H}, \mathrm{OCH}_{3}\right), 2.55-2.50\left(\mathrm{~m}, 4 \mathrm{H}, \mathrm{CH}_{2} \mathrm{CH}_{3} \times 2\right), 2.08-1.72\left(\mathrm{~m}, 4 \mathrm{H}, \mathrm{CH}_{2} \mathrm{CH}_{3} \times 2\right)$, 1.33-1.10 (m, 6H, $\left.\mathrm{CH}_{2} \mathrm{CH}_{3} \times 2\right), 0.80-0.64\left(\mathrm{~m}, 6 \mathrm{H}, \mathrm{CH}_{2} \mathrm{CH}_{3} \times 2\right)$. MS m/z $381\left(\mathrm{M}^{+-}\right)$. Anal. $\left(\mathrm{C}_{24} \mathrm{H}_{30} \mathrm{O}_{4}\right) \mathrm{C}, \mathrm{H}, \mathrm{O}$.

\section{3',3',5'-Tripropyl desmosdumotin C (25)}

$76 \%$ Yield. Yellow oil. ${ }^{1} \mathrm{H}$ NMR (300 MHz, $\left.\mathrm{CDCl}_{3}\right): \delta 19.29$ and $18.90(2: 1$, each s, $1 \mathrm{H}$, chelated-OH), 8.49 and $8.42(1: 2$, each d, $1 \mathrm{H}, J=15.6 \mathrm{~Hz}$, olefin), 7.95 and 7.92 (1:2, each d, $1 \mathrm{H}, J=15.6 \mathrm{~Hz}$, olefin), 7.74-7.65 (m, 2H, Ar-2,6-H), 7.44-7.34 (m, 3H, Ar-3,4,5-H), 4.00 and $3.92\left(2: 1\right.$, each s, $\left.3 \mathrm{H}, \mathrm{OCH}_{3}\right), 2.56-2.42\left(\mathrm{~m}, 2 \mathrm{H}, 5^{\prime}-\mathrm{CH}_{2} \mathrm{Et}\right), 2.00-1.65\left(\mathrm{~m}, 4 \mathrm{H}, 3^{\prime}-\right.$ $\left.\mathrm{CH}_{2} \mathrm{Et} \times 2\right), 1.65-1.48\left(\mathrm{~m}, 2 \mathrm{H}, 3^{\prime}-\mathrm{CH}_{2} \mathrm{CH}_{2} \mathrm{CH}_{3}\right), 1.16-0.96\left(\mathrm{~m}, 7 \mathrm{H}, 5^{\prime}-\mathrm{CH}_{2} \mathrm{CH}_{2} \mathrm{CH}_{3} \times 2\right.$ and 3'$\left.\mathrm{CH}_{2} \mathrm{CH}_{2} \mathrm{CH}_{3}\right), 0.90-0.77$ (m, 6H, 3'- $\left.\mathrm{CH}_{2} \mathrm{CH}_{2} \mathrm{CH}_{3} \times 2\right)$. MS m/z $395\left(\mathrm{M}^{+}-1\right)$. Anal. $\left(\mathrm{C}_{25} \mathrm{H}_{32} \mathrm{O}_{4}\right) \mathrm{C}, \mathrm{H}, \mathrm{O}$.

\section{4-Bromo-3',3',5'-tripropyl desmosdumotin C (26)}

$38 \%$ Yield. Yellow prisms, mp $114-115^{\circ} \mathrm{C} .{ }^{1} \mathrm{H}$ NMR (300 MHz, $\left.\mathrm{CDCl}_{3}\right): \delta 19.28$ and 18.81 (2:1, each $\mathrm{s}, 1 \mathrm{H}$, chelated-OH), 8.48 and $8.40(1: 2$, each $\mathrm{d}, 1 \mathrm{H}, J=15.6 \mathrm{~Hz}$, olefin), 7.86 and $7.84(1: 2$, each d, $1 \mathrm{H}, J=15.6 \mathrm{~Hz}$, olefin), 7.60-7.47 (m, $5 \mathrm{H}, \mathrm{Ar}-H), 4.00$ and 3.92 (2:1, each $\left.\mathrm{s}, 3 \mathrm{H}, \mathrm{OCH}_{3}\right), 2.58-2.41$ (m, 2H, 5'-CH $\left.{ }_{2} \mathrm{Et}\right), 2.00-1.80$ (m, 4H, 3'-CH $\left.\mathrm{H}_{2} \mathrm{Et} \times 2\right), 1.80-1.46$ (m, $\left.2 \mathrm{H}, 3^{\prime}-\mathrm{CH}_{2} \mathrm{CH}_{2} \mathrm{CH}_{3}\right), 1.18-0.93\left(\mathrm{~m}, 7 \mathrm{H}, 5^{\prime}-\mathrm{CH}_{2} \mathrm{CH}_{2} \mathrm{CH}_{3} \times 2\right.$ and $\left.3^{\prime}-\mathrm{CH}_{2} \mathrm{CH}_{2} \mathrm{CH}_{3}\right), 0.93-0.77$ (m, 6H, 3'- $\left.\mathrm{CH}_{2} \mathrm{CH}_{2} \mathrm{CH}_{3} \times 2\right)$. MS m/z 475 and $477\left(\mathrm{M}^{+}-1\right)$. Anal. $\left(\mathrm{C}_{25} \mathrm{H}_{31} \mathrm{O}_{4} \mathrm{Br}\right) \mathrm{C}, \mathrm{H}, \mathrm{O}$.

\section{Synthesis of Intermediates 31-36}

2-Acetyl-6-ethyl-3,5-dihydroxy-4,4-dimethylcyclohexa-2,5-dienone (31)-A solution of 2-acetyl-3,5-dihydroxy-4,4-dimethylcyclohexa-2,5-dienone (30,713 mg, 3.6 $\mathrm{mmol}$ ) and sodium methoxide $(2.0 \mathrm{~mL}, 9.3 \mathrm{mmol}, 25 \% \mathrm{MeOH}$ solution) in anhydrous $\mathrm{MeOH}$ $(3 \mathrm{~mL})$ containing ethyl iodide $(0.3 \mathrm{~mL}, 3.8 \mathrm{mmol})$ was refluxed for $4 \mathrm{~h}$. After removal of volatile solvent, the residue was partitioned between EtOAc and in aqueous $\mathrm{HCl}$. The water phase was extracted with EtOAc ( $\times 3)$. The combined organic layers were dried over $\mathrm{Na}_{2} \mathrm{SO}_{4}$ 
and concentrated in vacuo. The residue was chromatographed on silica gel with EtOAc-hexane ( $1: 9$ to $1: 4, \mathrm{v} / \mathrm{v}$ ) as an eluent to provide $\mathbf{3 1}(196 \mathrm{mg}, 24 \%)$ along with recovered starting material. Colorless prisms, mp 153-154 ${ }^{\circ} \mathrm{C}$ (EtOAc-hexane). ${ }^{1} \mathrm{H}$ NMR (300 MHz, DMSO-d 6 ): $\delta 19.00$ (br s, 1H, chelated-OH), 2.48 [s, 3H, C(O)CH $\mathrm{CH}_{3}, 2.35$ (q, 2H, J = 7.2 Hz, $\mathrm{CH}_{2} \mathrm{CH}_{3}$ ), 1.29 (s, $\left.6 \mathrm{H}, 4-\mathrm{CH}_{3} \times 2\right), 0.95\left(\mathrm{t}, 3 \mathrm{H}, 6-\mathrm{CH}_{2} \mathrm{CH}_{3}\right)$. MS m/z $223\left(\mathrm{M}^{+}-1\right)$. Anal. $\left(\mathrm{C}_{12} \mathrm{H}_{16} \mathrm{O}_{4} \cdot 1 / 8 \mathrm{H}_{2} \mathrm{O}\right) \mathrm{C}$, $\mathrm{H}, \mathrm{O}$.

2-Acetyl-4,4,6-triethyl-3,5-dihydroxycyclohexa-2,5-dienone (32)-A solution of 2,4,6-trihydroxyacetophenone $(1.117 \mathrm{~g}, 6.7 \mathrm{mmol})$ and sodium methoxide $(4.8 \mathrm{~mL}, 22.2 \mathrm{mmol}$, $25 \% \mathrm{MeOH}$ solution) in anhydrous $\mathrm{MeOH}(5 \mathrm{~mL})$ containing ethyl iodide $(1.65 \mathrm{~mL}, 20.6$ mmol) was refluxed for $7 \mathrm{~h}$. The reaction mixture was cooled to $0{ }^{\circ} \mathrm{C}$ and acidified with in aqueous $\mathrm{HCl}$, then extracted with EtOAc $(\times 3)$. The combined organic layers were dried over $\mathrm{Na}_{2} \mathrm{SO}_{4}$ and concentrated in vacuo. The residue was chromatographed on silica gel with EtOAc-hexane (1:9 to 1:4, v/v) as an eluent to provide $32(723 \mathrm{mg}, 43 \%)$. Colorless prisms, mp: $149-150{ }^{\circ} \mathrm{C}$ (EtOAc-hexane). ${ }^{1} \mathrm{H}$ NMR $(300 \mathrm{MHz}$, DMSO-d 6 ): $\delta 18.98$ (br s, $1 \mathrm{H}$, chelated$\mathrm{OH}), 2.51$ [s, 3H, C(O)CH $\mathrm{CH}_{3}$, $2.42\left(\mathrm{q}, 2 \mathrm{H}, J=7.2 \mathrm{~Hz}, 6-\mathrm{CH}_{2} \mathrm{CH}_{3}\right), 1.81(\mathrm{q}, 4 \mathrm{H}, J=7.4 \mathrm{~Hz}, 4-$ $\left.\mathrm{CH}_{2} \mathrm{CH}_{3} \times 2\right), 0.97\left(\mathrm{t}, 3 \mathrm{H}, J=7.2 \mathrm{~Hz}, 6-\mathrm{CH}_{3} \mathrm{CH}_{3}\right), 0.57\left(\mathrm{t}, 3 \mathrm{H}, J=7.2 \mathrm{~Hz}, 4-\mathrm{CH}_{2} \mathrm{CH}_{3} \times 2\right)$. MS $\mathrm{m} / \mathrm{z} 251\left(\mathrm{M}^{+}+1\right)$. Anal. $\left(\mathrm{C}_{21} \mathrm{H}_{22} \mathrm{O}_{4} \cdot 1 / 16 \mathrm{H}_{2} \mathrm{O}\right) \mathrm{C}, \mathrm{H}, \mathrm{O}$.

2-Acetyl-3,5-dihydroxy-4,4,6-tripropylcyclohexa-2,5-dienone (33)-The procedure was identical to that described above except with propyl iodide instead of ethyl iodide to provide 33 (41\%). Colorless prisms, mp: $95-96{ }^{\circ} \mathrm{C}$ (EtOAc-hexane). ${ }^{1} \mathrm{H}$ NMR (300 MHz, $\left.\mathrm{CD}_{3} \mathrm{OD}\right): \delta 2.53\left[\mathrm{~s}, 3 \mathrm{H}, \mathrm{C}(\mathrm{O}) \mathrm{CH}_{3}\right.$ ] $, 2.42\left(\mathrm{t}, 2 \mathrm{H}, J=7.5 \mathrm{~Hz}, 6-\mathrm{CH}_{2} \mathrm{Et}\right), 1.94-1.74(\mathrm{~m}, 4 \mathrm{H}, 4-$ $\left.\mathrm{CH}_{2} \mathrm{Et} \times 2\right), 1.54-1.38$ (m, 2H, , 6- $\mathrm{CH}_{3} \mathrm{CH}_{2} \mathrm{CH}_{3}$ ), 1.16-0.90 (m, 4H, 4- $\left.\mathrm{CH}_{3} \mathrm{CH}_{2} \mathrm{CH}_{3} \times 2\right), 0.94$ (t, $\left.3 \mathrm{H}, J=7.4 \mathrm{~Hz}, 6-\mathrm{CH}_{3} \mathrm{CH}_{2} \mathrm{CH}_{3}\right), 0.81\left(\mathrm{t}, 6 \mathrm{H}, J=7.1 \mathrm{~Hz}, 6-\mathrm{CH}_{3} \mathrm{CH}_{2} \mathrm{CH}_{3} \times 2\right)$. MS m/z 293 $\left(\mathrm{M}^{+}-1\right)$. Anal. $\left(\mathrm{C}_{17} \mathrm{H}_{26} \mathrm{O}_{4}\right) \mathrm{C}, \mathrm{H}, \mathrm{O}$.

2-Acetyl-6-ethyl-3-hydroxy-5-methoxy-4,4-dimethylcyclohexa-2,5-dienone (34) - To a solution of $\mathbf{3 1}(124 \mathrm{mg}, 0.55 \mathrm{mmol})$ in anhydrous EtOAc-MeOH $(5: 1,1.8 \mathrm{~mL})$, a solution of $\mathrm{TMSCHN}_{2}(2 \mathrm{~mL}, 4.0 \mathrm{mmol}, 2 \mathrm{M}$ solution in diethyl ether) was added slowly at $-78^{\circ} \mathrm{C}$ under argon atmosphere and the mixture was stirred for $3 \mathrm{~h}$. Acetic acid was then added to destroy the excess $\mathrm{TMSCHN}_{2}$. The mixture was concentrated in vacuo, and the residue was purified by silica gel column chromatography with EtOAc-hexane (1:9 to $1: 4 \mathrm{v} / \mathrm{v})$ as an eluent to obtain 34 (117 mg, 89\%). yellow oil. ${ }^{1} \mathrm{H}$ NMR $\left(300 \mathrm{MHz}, \mathrm{CDCl}_{3}\right)$ : $\delta 18.97$ and $18.14(2: 1$, each s, $1 \mathrm{H}$, chelated-OH), 3.97 and $3.90\left(2: 1\right.$, each s, $\left.3 \mathrm{H}, \mathrm{OCH}_{3}\right), 2.71$ and 2.61 [1:2, each s, $\left.3 \mathrm{H}, \mathrm{C}(\mathrm{O}) \mathrm{CH}_{3}\right], 2.48$ and $2.43\left(2: 1\right.$, each q, $\left.2 \mathrm{H}, J=7.4 \mathrm{~Hz}, 6-\mathrm{CH}_{2} \mathrm{CH}_{3}\right), 1.45$ and $1.34(1: 2$, each s, $\left.6 \mathrm{H}, 4-\mathrm{CH}_{3}\right), 1.16$ and $1.11\left(2: 1\right.$, each t, $\left.3 \mathrm{H}, 6-\mathrm{CH}_{2} \mathrm{CH}_{3} \times 2\right)$. MS m/z $237\left(\mathrm{M}^{+}-1\right)$.

\section{2-Acetyl-4,4,6-triethyl-3-hydroxy-5-methoxycyclohexa-2,5-dienone (35)-The} same procedure was employed to obtain $35(80 \%)$ from 32. yellow oil. ${ }^{1} \mathrm{H}$ NMR $(300 \mathrm{MHz}$, $\left.\mathrm{CDCl}_{3}\right): \delta 19.00$ and $18.12(3: 2$, each s, $1 \mathrm{H}$, chelated-OH), 4.02 and $3.93(3: 2$, each s, $3 \mathrm{H}$, $\left.\mathrm{OCH}_{3}\right), 2.71$ and $2.64\left[2: 3\right.$, each s, $\left.3 \mathrm{H}, \mathrm{C}(\mathrm{O}) \mathrm{CH}_{3}\right], 2.57$ and $2.51(3: 2$, each q, $2 \mathrm{H}, J=7.4 \mathrm{~Hz}$, 6- $\mathrm{CH}_{2} \mathrm{CH}_{3}$ ), 2.03-1.72 (m, 4H, 4- $\left.\mathrm{CH}_{2} \mathrm{CH}_{3} \times 2\right), 1.18$ and 1.12 (3:2, each t, $3 \mathrm{H}, 6-\mathrm{CH}_{2} \mathrm{CH}_{3}$ ), 0.67 and $0.65\left(2: 3\right.$, each t, $\left.6 \mathrm{H}, 4-\mathrm{CH}_{2} \mathrm{CH}_{3} \times 2\right)$. MS $m / z 267\left(\mathrm{M}^{+}+1\right)$.

2-Acetyl-3-hydroxy-5-methoxy-4,4,6-tripropylcyclohexa-2,5-dienone (36)-The same procedure was employed to obtain $\mathbf{3 6}(53 \%)$ from $\mathbf{3 3}$ along with recovered starting material (32\%). yellow oil. ${ }^{1} \mathrm{H}$ NMR (300 MHz, $\left.\mathrm{CDCl}_{3}\right)$ : $\delta 18.99$ and 18.15 (3:2, each s, $1 \mathrm{H}$, chelated-OH), 3.98 and $3.90\left(3: 2\right.$, each s, $\left.3 \mathrm{H}, \mathrm{OCH}_{3}\right), 2.67$ and 2.63 [2:3, each s, $3 \mathrm{H}, \mathrm{C}(\mathrm{O})$ $\mathrm{CH}_{3}$ ], 2.53-2.40 (m, 2H, 6- $\left.\mathrm{CH}_{2} \mathrm{Et}\right), 1.95-1.42\left(\mathrm{~m}, 6 \mathrm{H}, 6-\mathrm{CH}_{2} \mathrm{CH}_{2} \mathrm{CH}_{3}\right.$ and $\left.4-\mathrm{CH}_{2} \mathrm{Et} \times 2\right)$, 1.10-0.95 (m, 7H, 6- $\mathrm{CH}_{2} \mathrm{CH}_{2} \mathrm{CH}_{3}$ and 4- $\left.\mathrm{CH}_{2} \mathrm{CH}_{2} \mathrm{CH}_{3} \times 2\right), 0.83$ and 0.80 (3:2, each t, 6H, 4$\left.\mathrm{CH}_{2} \mathrm{CH}_{2} \mathrm{CH}_{3} \times 2\right)$. $\mathrm{MS} \mathrm{m} / 2,307\left(\mathrm{M}^{+-1}\right)$. 


\title{
Cytotoxic Activity Assay 15
}

All stock cultures were grown in T-25 flasks. Freshly trypsinized cell suspensions were seeded in 96-well microtitre plates at densities of 1500-7500 cells per well with compounds added from DMSO-diluted stock. After 3 days in culture, attached cells were fixed with cold 50\% trichloroacetic acid and then stained with $0.4 \%$ sulforhodamine B. The absorbency at $562 \mathrm{~nm}$ was measured using a microplate reader after solubilizing the bound dye. The mean $\mathrm{ED}_{50}$ is the concentration of agent that reduces cell growth by $50 \%$ under the experimental conditions and is the average from at least three independent determinations that were reproducible and statistically significant. The following human tumor cell lines were used in the assay: A549 (human lung carcinoma), A431 (epidermoid skin carcinoma), 1A9 (human ovarian carcinoma), HCT-8 (colon adenocarcinoma), PC-3 (prostate cancer), KB (nasopharyngeal carcinoma), KBVTN (vincristine-resistant KB subline), HUVEC (human endothelial). All cell lines were obtained from the Lineberger Cancer Center (UNC-CH) or from ATCC (Rockville, MD) and were cultured in RPMI-1640 medium supplemented with 25 mM HEPES, $0.25 \%$ sodium bicarbonate, $10 \%$ fetal bovine serum, and $100 \mu \mathrm{g} / \mathrm{mL}$ kanamycin.

\section{Angiogenesis Assay}

Method is according to the NCI's Angiogenesis Resource Center protocol. HUVEC were purchased from Cambrex Bio-science. HUVEC $\left(1.5 \times 10^{3}\right)$ were plated in a 96-well plate in $100 \mu \mathrm{l}$ of EGM-2 (Clonetec $H$ CC3162). After $24 \mathrm{~h}$ (day zero), the test compound (100 $\mu \mathrm{l}$ ) was added to each well at $2 \times$ the desired concentration in EGM- 2 medium. One plate was stained with $0.5 \%$ crystal violet in $20 \% \mathrm{MeOH}$ for 10 minutes, rinsed with water, and air-dried. The remaining plates were incubated for $72 \mathrm{~h}$ at $37{ }^{\circ} \mathrm{C}$. After $72 \mathrm{~h}$, plates were stained with $0.5 \%$ crystal violet in $20 \% \mathrm{MeOH}$, rinsed with water and air-dried. The stain was eluted with 1:1 solution of EtOH:0.1 M sodium citrate, and absorbance was measured at $540 \mathrm{~nm}$ with an ELISA reader.

\section{Supplementary Material}

Refer to Web version on PubMed Central for supplementary material.

\section{Acknowledgements}

This work was supported by a NIH grant CA17625 from the National Cancer Institute, awarded to K. H. Lee.

\author{
Abbreviations \\ HUVEC \\ human umbilical vein endothelial cell \\ KB \\ vincristine-resistant \\ KB-VIN \\ expressing P-glycoprotein, cell subline

\section{References} \\ 1. Balunas MJ, Kinghorn AD. Drug discovery from medicinal plants. Life Science 2005;78:431-441. \\ 2. Rollinger JM, Langer T, Stuppner H. Strategies for efficient lead structure discovery from natural \\ products. Curr Med Chem 2006;13:1491-1507. [PubMed: 16787200] \\ 3. Jones WP, Chin YW, Kinghorn AD. The role of pharmacognosy in modern medicine and pharmacy. \\ Curr Drug Targets 2006;7:247-264. [PubMed: 16515526]
}


4. Butler MS. Natural products to drugs: natural product derived compounds in clinical trials. Nat Prod Rep 2005;22:162-195. [PubMed: 15806196]

5. Koehn FE, Carter GT. The evolving role of natural products in drug discovery. Nat Rev Drug Discov 2005;4:206-220. [PubMed: 15729362]

6. Paterson I, Anderson EA. The renaissance of natural products as drug candidates. Science 2005;310:451-53. [PubMed: 16239465]

7. Tan G, Gyllenhaal C, Soejarto DD. Biodiversity as a source of anticancer drugs. Curr Drug Targets 2006;7:265-277. [PubMed: 16515527]

8. http://www.who.int/mediacentre/factsheets/fs297/en/

9. Jiang Su New Medical College, Ed.. Chung Yao Da Tzu Dien (Dictionary of Chinese Materia Medicia). 2. Shanghai Science \& Technology Press; Hong Kong: 1977. p. 1919

10. Wu JH, McPhail AT, Bastow KF, Shiraki H, Ito J, Lee KH. Tetrahedron Lett 2002;43:1391-1393.

11. Nakagawa-Goto K, Wu JH, Lee KH. First total synthesis of desmosdumotin C. Syn Commun 2005;35:1735-1739.

12. Nakagawa-Goto K, Wu JH, Bastow KF, Wu CC, Lee KH. Antitumor agents 243. Syntheses and cytotoxicity of desmosdumotin C derivatives. Bioorg Med Chem 2005;13:2325-2330. [PubMed: 15727881]

13. Bick IRC, Horn DHD. Nuclear magnetic resonance studies. V. The tautomerism of tasmanone and related $\beta$-triketones. Aust J Chem 1965;18:1405-1410.

14. a) Wollenweber E, Dietz VH, Schilling G, Favre-Bonvin J, Smith DM. Flavonoids from chemotypes of the goldback fern, Pityrogramma tiangularis. Phytochemistry 1985;24:965-972. b) Sbit M,

Dupont L, Dideberg O, Vilain C. Structure of ceroptene. Acta Crystallogr Sect C 1987;C43:22042206.

15. Nakanishi Y, Chang FR, Liaw CC, Wu YC, Bastow KF, Lee KH. Acetogenins as selective inhibitors of the human ovarian 1A9 tumor cell line. J Med Chem 2003;46:3185. [PubMed: 12852747] 


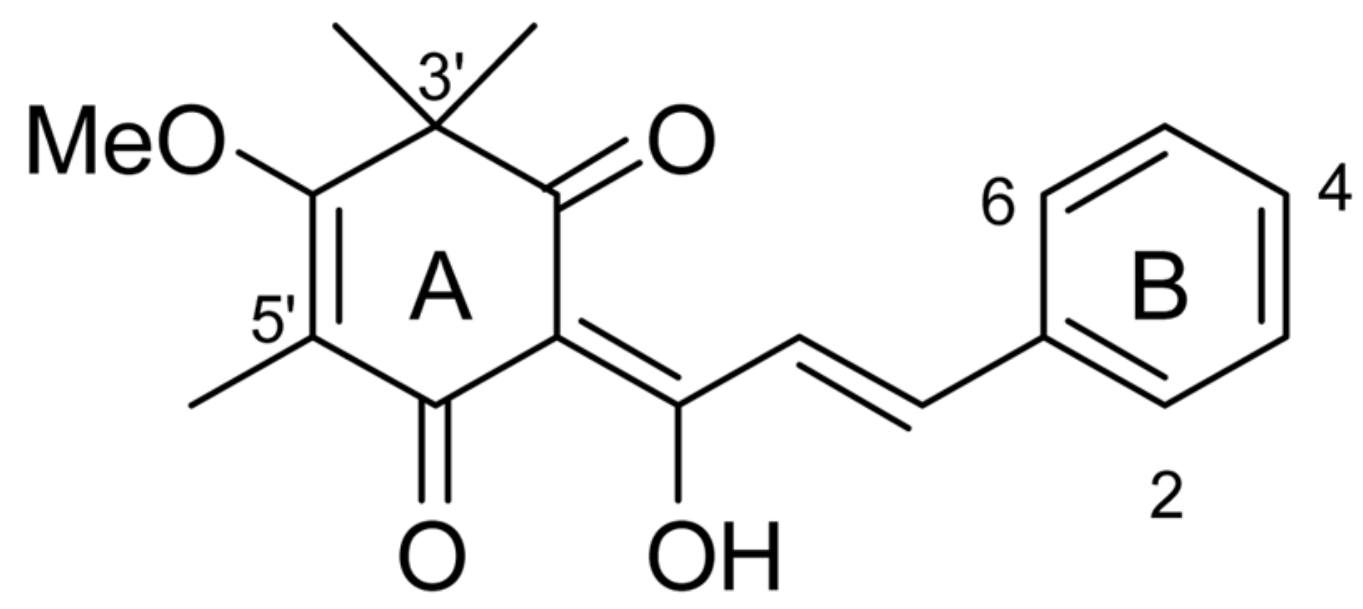

1 Desmosdumotin C

Figure 1.

Structure of $\mathbf{1}$ 
<smiles>Oc1cc(O)c(Cl)c(Cl)c1</smiles>

28<smiles>COC1=C(C)C(=O)C(C(C)(C)C)=C(O)C1(C)C</smiles>

29

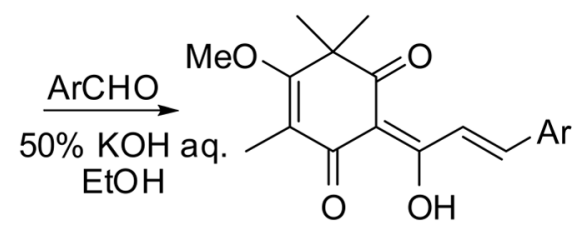

1-18

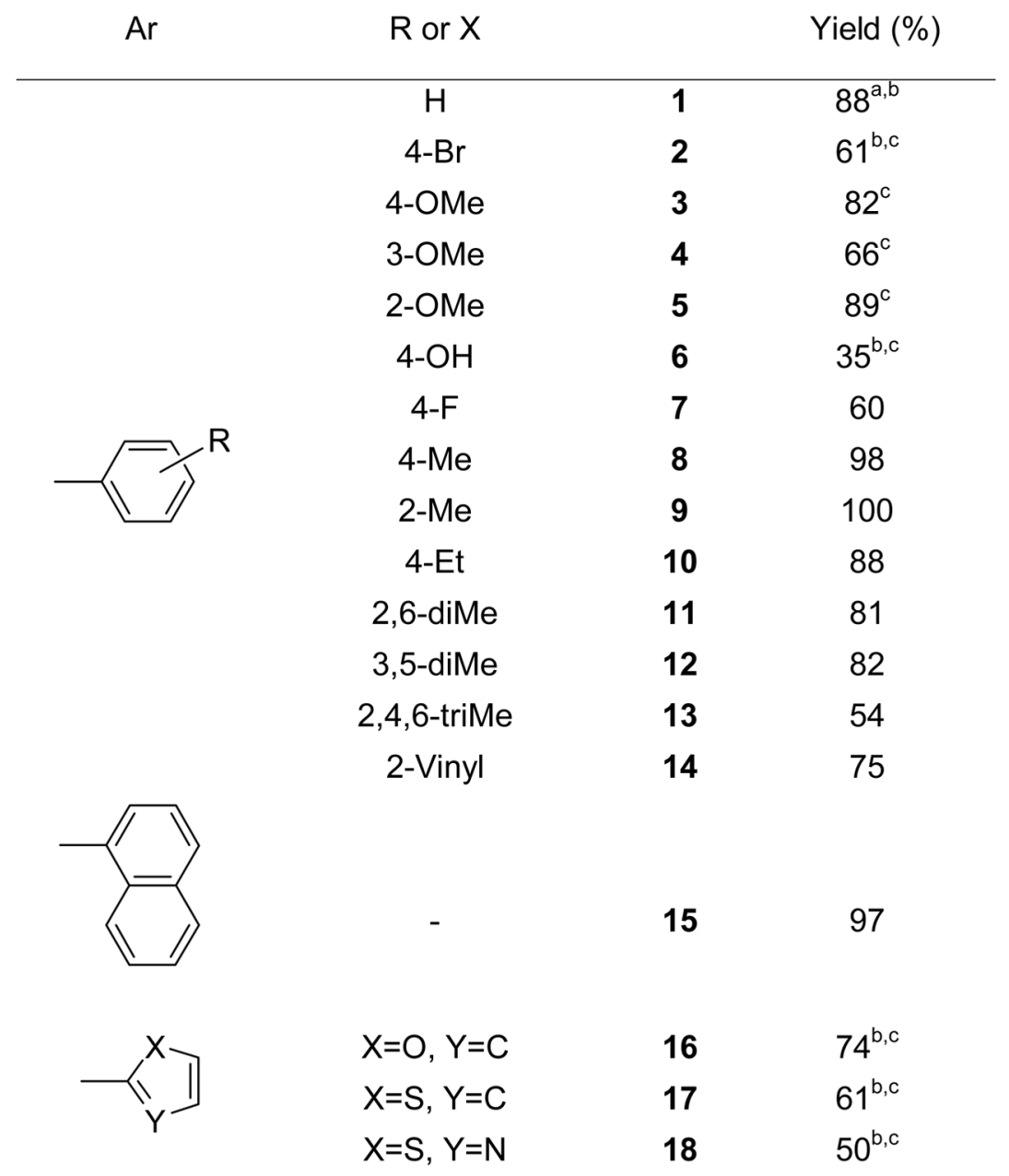

Scheme 1.

Syntheses of 1-Analogs: B-ring Modifications

a See reference $11 .{ }^{b}$ Based on recovered starting material. ${ }^{\mathrm{c}}$ See reference ${ }^{12}$. 


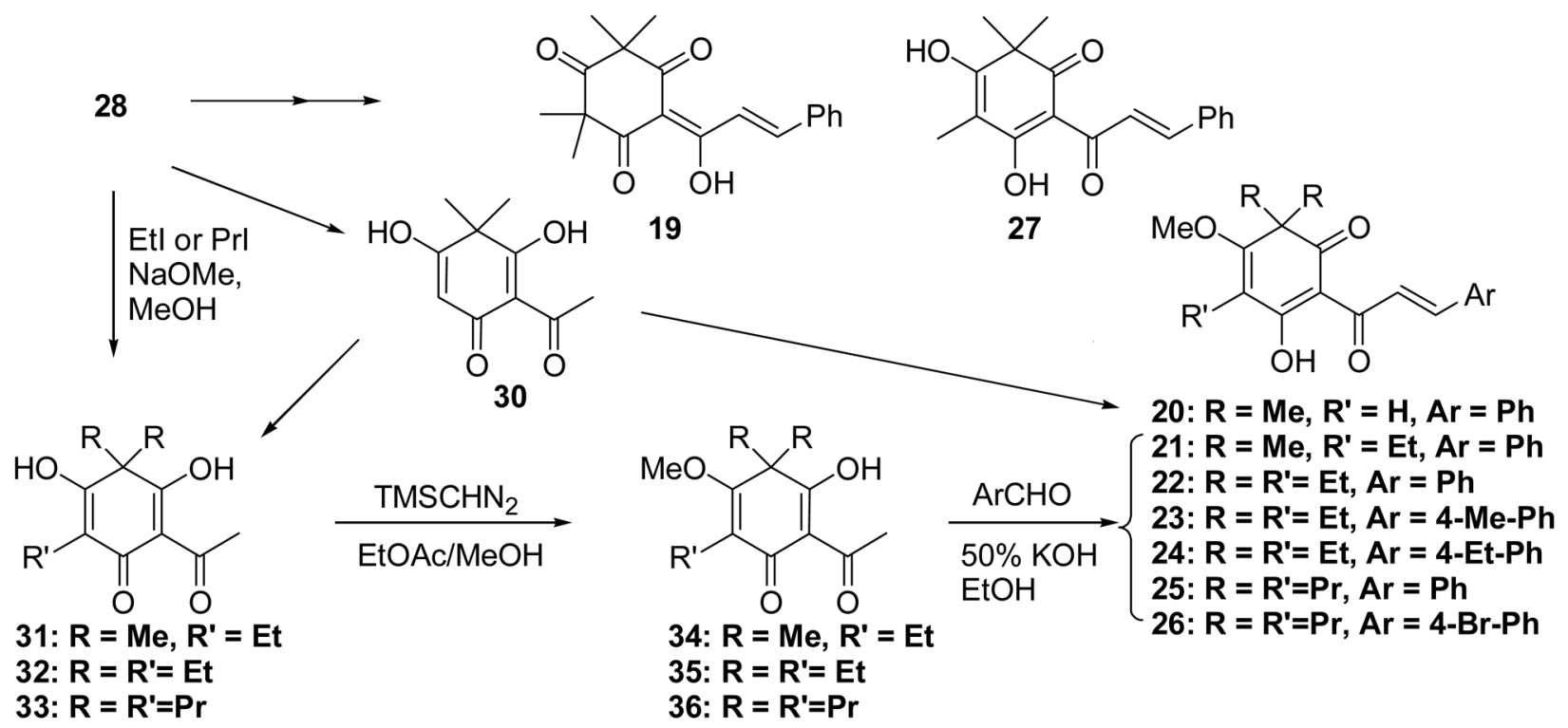

Scheme 2.

Syntheses of 1-Analogs: A/B-ring Modifications 


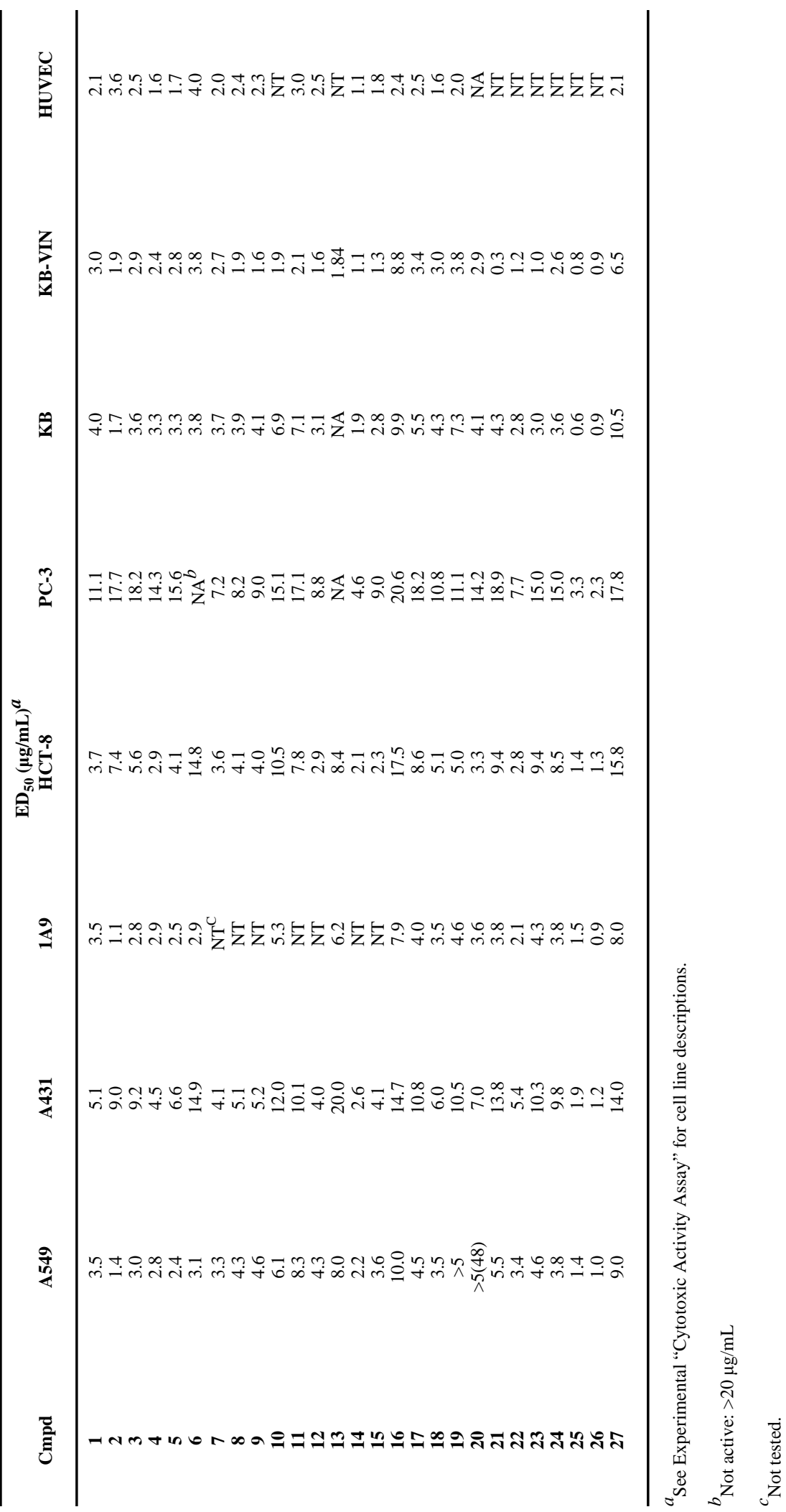

